\title{
Role of extracorporeal cardiopulmonary resuscitation in adults
}

\author{
Hongsun Kim, Yang Hyun Cho \\ Department of Thoracic and Cardiovascular surgery, Samsung Medical Center, Sungkyunkwan University School of Medicine, Seoul, Korea
}

Extracorporeal cardiopulmonary resuscitation (ECPR) has been performed with increasing frequency worldwide to improve the low survival rate of conventional cardiopulmonary resuscitation (CCPR). Several studies have shown that among patients who experience in-hospital cardiac arrest, better survival outcomes and neurological outcomes can be expected after ECPR than after CCPR. However, studies have not clearly shown a short-term survival benefit of ECPR for patients who experience out-of-hospital cardiac arrest. Favorable outcomes are associated with a shorter low-flow time, an initial shockable rhythm, lower serum lactate levels, higher blood pH, and a lower Sequential Organ Failure Assessment score. Indications for ECPR include young age, witnessed arrest with bystander cardiopulmonary resuscitation, an initial shockable rhythm, correctable causes such as a cardiac etiology, and no return of spontaneous circulation within 10-20 minutes of CCPR. ECPR is a complex intervention that requires a highly trained team, specialized equipment, and multidisciplinary support within a healthcare system, and it has the risk of several life-threatening complications. Therefore, physicians should carefully select patients for ECPR who can gain the most benefit, instead of applying ECPR indiscriminately.

Key Words: advanced cardiac life support; extracorporeal membrane oxygenation; cardiopulmonary resuscitation; out-of-hospital cardiac arrest

\section{INTRODUCTION}

Despite numerous studies and detailed guidelines, survival after cardiopulmonary resuscitation for sudden cardiac arrest remains low. Reported survival rates range from $15 \%$ to $17 \%$ for in-hospital cardiac arrest (IHCA) and from $8 \%$ to $10 \%$ for out-of-hospital cardiac arrest (OHCA) [1-3]. It is possible that more robust end-organ support is the key to improving survival rather than chest compressions and endotracheal ventilation. Extracorporeal life support (ECLS) is a life support system that provides artificial extracorporeal blood oxygenation and circulatory support. Venoarterial ECLS uses the principles of cardiopulmonary bypass, implementing a blood pump and artificial lung to replace natural cardiopulmonary function. It was first proposed for cardiac resuscitation in the early 1960s [4]. After subsequent progression in ECLS technology, now it is deployed rapidly for patients in cardiac arrest.

\section{Concept and Definition of Extracorporeal Cardiopulmonary Resuscitation}

The goal of extracorporeal cardiopulmonary resuscitation (ECPR) is to support end-organ

\section{Review Article}

Received: February 25, 2020

Accepted: February 27, 2020

Corresponding author

Yang Hyun Cho

Department of Thoracic and

Cardiovascular Surgery, Samsung

Medical Center, Sungkyunkwan

University School of Medicine,

81 Irwon-ro, Gangnam-gu, Seoul

06351, Korea

Tel: +82-2-3410-2213

Fax: +82-2-3410-0089

E-mail:mdcho95@gmail.com

Copyright (@) 2020 The Korean Society of Critical Care Medicine

This is an Open Access article distributed under the terms of Creative Attributions Non-Commercial License (https:// creativecommons.org/li-censes/by-nc/4.0/) which permits unrestricted noncommercial use, distribution, and reproduction in any medium, provided the original work is properly cited. 
perfusion while potentially reversible conditions are addressed. It is defined as initiation of ECLS during cardiopulmonary resuscitation after conventional measures have failed or after repetitive arrest events have occurred without return of spontaneous circulation (ROSC) for $>20$ minutes [5]. The general concept is to replace conventional cardiopulmonary resuscitation (CCPR) with ECLS. However, ECPR is a much more complex intervention and requires a highly trained team, specialized equipment, multidisciplinary support, and setup time ranging between 5 and 30 minutes (Figure 1).

It is difficult to determine ROSC once ECPR has been initiated. In CCPR, patient vital signs are regularly monitored to determine ROCS. In contrast, the ECLS system is not routinely stopped during ECPR to check: this is why the textbook definition cited above specifies "repetitive arrest without ROSC for $>20$ minutes." Based on this definition, ECLS initiated immediately after ROSC can be considered ECPR. Because sustained ROSC is important in defining successful resuscitation, direct comparison of ECPR and CCPR is difficult and confusing to both researchers and clinicians. Understanding the differences between these two methods is crucial to properly interpret ECPR studies.

Since 1989, the Extracorporeal Life Support Organization (ELSO) has collected ECLS usage and outcome data. According to the ELSO registry, the incidence of ECPR use increased more than 10-fold between 2003 and 2014, from 35 to over 400 per year (Figure 2) [6,7], and the overall survival to hospital discharge was $29 \%$ in adults [8]. The Advanced Cardiac Life Support (ACLS) Guidelines state that ECPR may be considered for selected patients as rescue therapy when CCPR efforts are failing in settings in which it can be expeditiously implemented and supported by skilled providers (class $2 b$; level of evidence, C-LD) $[9,10]$. However, there is insufficient evidence to recommend its routine use in patients with cardiac arrest. This review focuses on the current evidence for ECPR indications and the benefit of ECPR over CCPR.

\section{KEY MESSAGES}

- For in-hospital cardiac arrest, extracorporeal cardiopulmonary resuscitation (ECPR) provides better survival and neurological outcomes than conventional cardiopulmonary resuscitation (CCPR).

- For out-of-hospital cardiac arrest, ECPR provides better medium-term survival outcomes and overall neurological outcomes than CCPR.

- A short low-flow time and an initial shockable rhythm are associated with better outcomes of ECPR.

\section{CURRENT EVIDENCE}

\section{ECPR versus CCPR}

To date, there have been no large prospective randomized controlled trials (RCTs) comparing ECPR and CCPR. However,

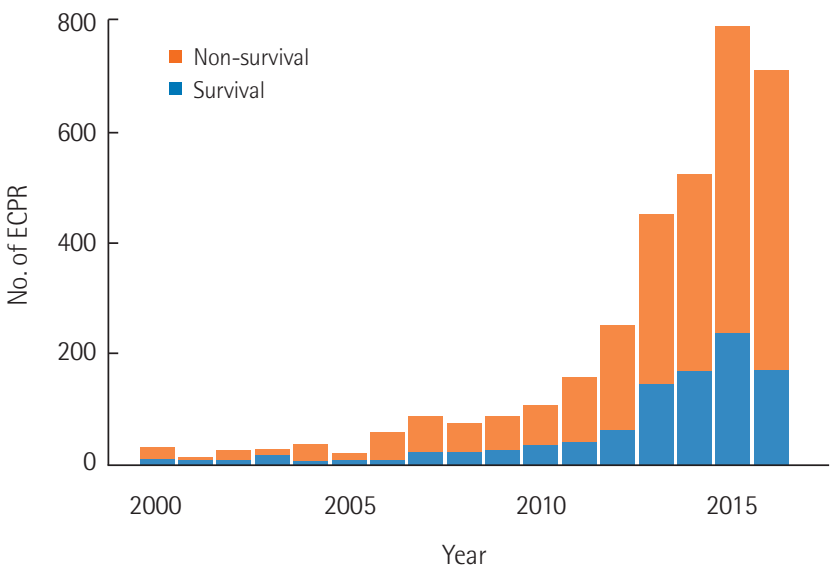

Figure 2. Annual extracorporeal cardiopulmonary resuscitation (ECPR) episodes 2000-2016 [6]. According to Extracorporeal Life Support Organization registry, the number of ECPR has increased worldwide, and so has the survival rate. The recent reported survival to discharge was $23 \%$ to $31 \%$.

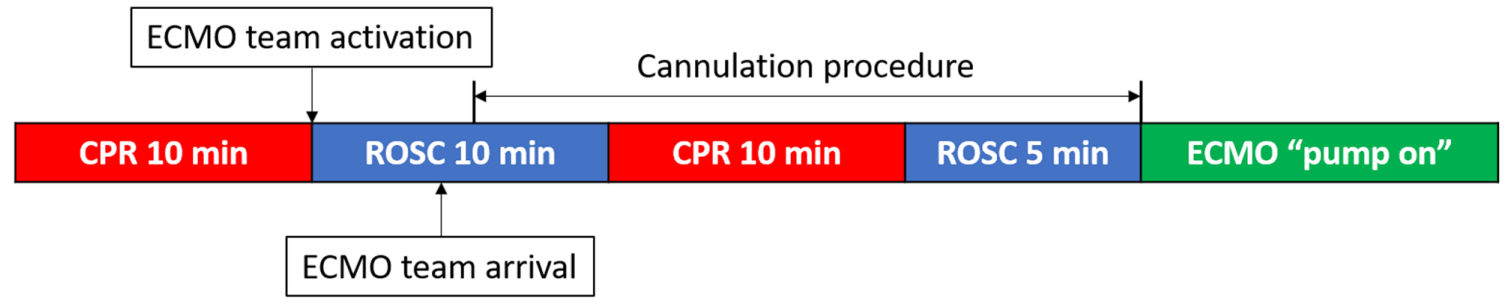

Figure 1. A typical example of in-hospital extracorporeal cardiopulmonary resuscitation (ECPR). There was two recovery of spontaneous circulation (ROSC) during whole CPR process. Extracorporeal circulation was successfully established after 35 minutes of resuscitation which includes 20 minutes of convectional resuscitation and 15 minutes of ROSC. ECPR is defined, despite pump-on during ROSC, if ROSC duration is less than 20 minutes. Because resuscitation is complex and various, like this example, there can be debates on this definition of ECPR. ECMO: extracorporeal membrane oxygenation. 
numerous ECPR studies have resulted in several notable findings (Table 1).

\section{Overall Survival to Discharge}

A subgroup meta-analysis of 4 propensity score-matched studies that compared overall survival to discharge between ECPR and CCPR by Kim et al. [11] showed no significant difference; however, there was a nonsignificant trend toward benefit of ECPR for overall survival to discharge (risk ratio [RR], 1.86; $95 \%$ confidence interval [CI], 0.99 to $3.50 ; \mathrm{P}=0.05$ ). In another meta-analysis of 17 studies by Twohig et al. [12], subgroup analysis of nine propensity score-matched studies found a significant benefit of ECPR over CCPR for overall survival at discharge or 30 days (odds ratio [OR], 0.40; 95\% CI, 0.27 to 0.60 ; $\mathrm{P}<0.001)$. However, there was moderate heterogeneity $(\mathrm{P}=0.01$, $\mathrm{I}^{2}=59 \%$ ) among the studies. According to these results, the deployment of ECPR tends to improve survival to discharge compared with CCPR. However, the outcomes according to type of cardiac arrest should be analyzed and reviewed carefully due to study heterogeneity. In addition and more importantly, high possibility of cardiac origin and reversibility was the indication for ECPR in most of the studies. Furthermore, these studies had two major limitations that should be considered in their interpretation. First, no study disclosed how cardiac cause and reversibility was suspected in the ECPR groups. Second, the control groups (CCPR) were generally unselected, as CCPR is not selectively performed. This selection bias between ECPR and CCPR patients cannot be adjusted for by regression analysis or propensity score matching.

\section{Outcome in IHCA}

Chen et al. [13] reported an observation study comprised of 172 witnessed IHCAs that found a short-term and long-term survival benefit for ECPR over CCPR. Additionally, neurological outcomes tended to be better at discharge for ECPR patients (30.4\% vs. $15.2 \%, P=0.09)$. In 2011 , another retrospective single center study investigated 120 IHCA patients and showed that ECPR was associated with a survival benefit over CCPR in patients who received CPR for more than 10 minutes, especially in patients with a cardiac origin [14]. The study also showed better neurological outcome in patients who received ECPR.

Table 1. Previously reported survival and neurological outcome comparisons of ECPR and CCPR

\begin{tabular}{|c|c|c|c|c|}
\hline Study & Type of arrest & outcome & ECPR vs. CCPR & P-value \\
\hline \multirow[t]{5}{*}{ Chen et al. (2008) [13] } & \multirow[t]{5}{*}{ IHCA } & Survival rate to discharge & $\mathrm{HR}, 0.51 ; 95 \% \mathrm{Cl}, 0.35-0.74$ & $<0.001$ \\
\hline & & Survival at 30 days & $\mathrm{HR}, 0.47 ; 95 \% \mathrm{Cl}, 0.28-0.77$ & 0.003 \\
\hline & & Survival at 1 year & $\mathrm{HR}, 0.53 ; 95 \% \mathrm{Cl}, 0.33-0.83$ & 0.006 \\
\hline & & CPC score $1-2$ at discharge & $30.4 \%$ vs. $15.2 \%$ & 0.09 \\
\hline & & CPC score $1-2$ at 1 year & $19.5 \%$ vs. $10.8 \%$ & 0.27 \\
\hline \multirow[t]{8}{*}{ Shin et al. (2011) [14] } & \multirow[t]{8}{*}{ IHCA } & Survival to discharge & $31.7 \%$ vs. $10.0 \%$ & 0.013 \\
\hline & & Cardiac origin & $35.5 \%$ vs. $8.8 \%$ & 0.004 \\
\hline & & Survival at 6 months & $26.7 \%$ vs. $8.3 \%$ & 0.019 \\
\hline & & Cardiac origin & $28.9 \%$ vs. $8.9 \%$ & 0.035 \\
\hline & & CPC score $1-2$ at discharge & $23.3 \%$ vs. $5.0 \%$ (adjusted $\mathrm{OR}, 0.17 ; 95 \% \mathrm{Cl}, 0.04-0.68$ ) & 0.012 \\
\hline & & Cardiac origin & $26.7 \%$ vs. $8.8 \%$ (adjusted $\mathrm{OR}, 0.19 ; 95 \% \mathrm{Cl}, 0.04-0.82$ ) & 0.026 \\
\hline & & CPC score $1-2$ at 6 months & $23.3 \%$ vs. $5.0 \%(\mathrm{HR}, 0.48 ; 95 \% \mathrm{Cl}, 0.29-077)$ & 0.003 \\
\hline & & Cardiac origin & $26.7 \%$ vs. $6.7 \%(\mathrm{HR}, 0.56 ; 95 \% \mathrm{Cl}, 0.33-0.97)$ & 0.038 \\
\hline \multirow[t]{3}{*}{ Maekawa et al. (2013) [15] } & \multirow[t]{3}{*}{$\mathrm{OHCA}$} & Survival to discharge & $37.5 \%$ vs. $12.5 \%$ & 0.093 \\
\hline & & Survival at 3 months & $37.5 \%$ vs. $8.3 \%$ & 0.036 \\
\hline & & CPC score $1-20$ at 3 months & $29.2 \%$ vs. $8.3 \%$ & 0.14 \\
\hline \multirow[t]{4}{*}{ Kim et al. (2014) [16] } & \multirow[t]{4}{*}{$\mathrm{OHCA}$} & Survival at 24 hours & $57.5 \%$ vs. $30.8 \%$ & 0.010 \\
\hline & & Survival to discharge & $17.3 \%$ vs. $21.2 \%$ & 0.804 \\
\hline & & Survival at 3 months & $15.4 \%$ vs. $7.7 \%$ & 0.358 \\
\hline & & CPC score $1-2$ at 3 months & $15.4 \%$ vs. $1.9 \%$ & 0.031 \\
\hline
\end{tabular}

ECPR: extracorporeal cardiopulmonary resuscitation; CCPR: conventional cardiopulmonary resuscitation; IHCA: in-hospital cardiac arrest; HR, hazard ratio; $\mathrm{Cl}$, confidence interval; $\mathrm{CPC}$ : cerebral performance category; $\mathrm{OR}$, odds ratio. 
According to a meta-analysis of three retrospective propensity score-matched studies comparing ECPR and CCPR in IHCA, ECPR was associated with better survival at discharge (RR, 2.37; 95\% CI, 1.35 to $4.15 ; \mathrm{P}=0.003$ ) and at 3-6 months $(\mathrm{RR}, 2.54 ; 95 \% \mathrm{CI}, 1.38$ to $4.66 ; \mathrm{P}=0.003$ ) (Figure 3 ) [11]. Moreover, the study reported better neurological outcome (cerebral performance category [CPC] score 1-2) at discharge in the ECPR group (RR, 2.72; 95\% CI, 1.21 to $6.13 ; \mathrm{P}=0.02$ ). Although no high-quality evidence is currently available, the studies so far have shown better survival and neurological outcomes in IHCA patients who receive ECPR.

\section{Outcome in OHCA}

According to a post hoc analysis of a prospective observational cohort reported in Japan [15], 3-month survival of OHCA patients was higher in the matched ECPR group than the matched CCPR group (37.5\% vs. 8.3\%, $\mathrm{P}=0.036$; stratified log-rank $\mathrm{P}=0.018$ ). However, the study did not find a significant difference in survival rate at discharge or neurological outcome between ECPR and CCPR.

In contrast, a multivariate retrospective analysis of a prospective cohort that included 499 patients showed that ECPR should be considered in order to achieve a good neurological outcome in OHCA patients who required prolonged CPR, especially $\geq 21$ minutes [16]. However, there was no significant difference in survival to discharge or 3-month survival between ECPR and CCPR.
Kim et al. [11] conducted a meta-analysis comparing ECPR and CCPR in OHCA that included the above two observational studies. Although the analysis showed no significant difference in survival at discharge (RR, 1.45; 95\% CI, 0.41 to 5.16; $\mathrm{P}=0.56$ ), ECPR was associated with significantly better survival at 3-6 months (RR, 2.74; 95\% CI, 1.13 to $6.67 ; \mathrm{P}=0.03$ ) and better neurological outcome at 3 months (RR, 4.64; 95\% CI, 1.41 to $15.25 ; \mathrm{P}=0.01$ ) (Figure 3 ) [11]. Unlike IHCA, there is a lack of evidence to support a benefit in survival at discharge for ECPR over CCPR in patients with OHCA. On the other hand, studies have shown that the use of ECPR in OHCA improves both medium-term survival (3-6 months) and neurological outcome. We believe that the ECPR patients in OHCA were less selected than those in IHCA because of lack of information and lack of time to investigate in the emergency room.

\section{Prognostic Factors in ECPR}

Prognostic factors for ECPR largely overlap with those for CCPR. Among 133 patients who received ECPR in a retrospective study, low-flow time (duration of CCPR until perfusion of ECPR started) strongly correlated with survival $(\mathrm{P}<0.001)$ and was an independent predictor of mortality [17]. Similarly, another retrospective analysis of 111 ECPR patients showed that survival to discharge was associated with shorter CPR duration $(\mathrm{P}=0.022)$ and younger age $(\mathrm{P}=0.003)[18]$.

Neurologic outcome of ECPR according to the initial cardiac arrest heart rhythm has been evaluated in several studies [16,

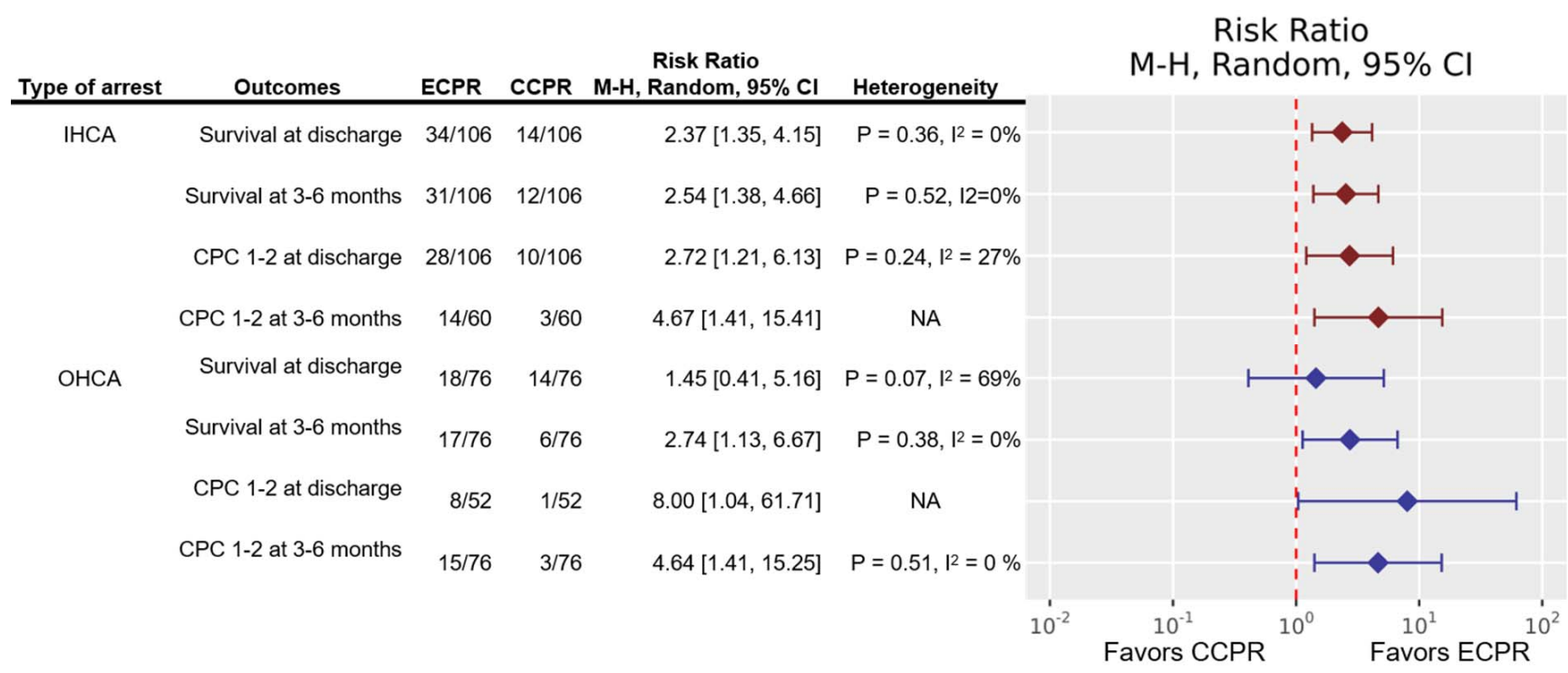

Figure 3. Results reported by the meta-analysis by Kim et al. [11]. Risk ratios and 95\% confidence intervals (Cls) were plotted in logarithmic scale. All outcomes, except for survival at discharge in out-of-hospital cardiac arrest (OHCA), favor extracorporeal cardiopulmonary resuscitation (ECPR), although there was a little in-study heterogeneity (red: IHCA, blue: OHCA). CCPR: conventional cardiopulmonary resuscitation; IHCA: in-hospital cardiac arrest; CPC: cerebral performance category; NA: not applicable. 
19]. A retrospective observational study of 294 adult patients who underwent ECPR for IHCA found that poor neurological outcome (CPC score 3-5) was associated with the following initial heart rhythms: asystole, 88.1\%; pulseless electrical activity (PEA), 66.3\%; and shockable rhythm, 50.6\% ( $\mathrm{P}<0.001)$. In addition, a significant association was found between lowflow time and neurologic outcome in patients with initial PEA $(\mathrm{P}=0.005)$ and/or shockable rhythm $(\mathrm{P}=0.006)$ [19]. Another retrospective analysis based on a prospective cohort of 499 adults who experienced OHCA showed that neurologic outcome in patients who received ECPR correlated with age $(\mathrm{P}=0.014)$, witnessed arrest without initial asystole $(\mathrm{P}=0.015)$, mean arterial pressure $>60 \mathrm{~mm}$ Hg within 2 hours after ECPR $(P=0.021)$, left ventricular ejection fraction after ECPR implantation $(P=0.009)$, therapeutic hypothermia $(P=0.025)$, and complications during ECPR $(\mathrm{P}=0.047)[16]$. In a retrospective observational study of 95 adult patients who underwent ECPR during hospitalization, Ryu et al. [20] proposed a new risk prediction model for neurologic outcome (C-statistic, 0.867; 95\% $\mathrm{CI}, 0.823$ to $0.912, \mathrm{P}=0.917$ ) using age, initial Sequential Organ Failure Assessment (SOFA) score, first monitored arrest rhythm, duration of low-flow time, initial pulse pressure, initial mean arterial pressure, and serum glucose level as independent variables [20].

According to a meta-analysis of 856 patients who underwent ECPR following IHCA pooled from 11 studies, survival correlated with initial shockable rhythm (OR, 1.65; 95\% CI, 1.05 to $2.61 ; \mathrm{P}=0.03$ ), shorter low-flow time (pooled mean dif- ference [PMD], -17.15 minutes; 95\% CI, -20.90 to -13.40 minutes; $\mathrm{P}<0.001)$, lower lactate level both immediately before ECPR start (PMD, $-4.12 \mathrm{mmol} / \mathrm{L} ; 95 \% \mathrm{CI},-6.0$ to $-2.24 \mathrm{mmol} / \mathrm{L}$; $\mathrm{P}<0.001$ ) and on intensive care unit (ICU) admission (PMD, $-4.13 \mathrm{mmol} / \mathrm{L} ; 95 \% \mathrm{CI},-6.38$ to $-1.88 \mathrm{mmol} / \mathrm{L}$; $\mathrm{P}<0.001)$, lower SOFA score (PMD, $-1.71 ; 95 \% \mathrm{CI},-2.93$ to $-0.50 ; \mathrm{P}=0.006$ ), and lower creatinine level within 24 hours after ICU admission (PMD, $-0.37 \mathrm{mg} / \mathrm{dL} ; 95 \% \mathrm{CI},-0.54$ to $-0.19 \mathrm{mg} / \mathrm{dL} ; \mathrm{P}<0.001$ ) [21]. However, the study did not find a significant association between survival and age, sex, or cardiac vs. non-cardiac etiology.

Similarly, a meta-analysis of 15 primary studies that included a total of 841 patients who underwent ECPR for OHCA showed that favorable outcome, defined as survival with CPC score 1-2 at 30 days or at discharge, was significantly associated with low-flow duration $(\mathrm{P}=0.04)$, initial shockable rhythm $(\mathrm{OR}, 2.20$; 95\% CI, 1.30 to $3.72 ; \mathrm{P}=0.003)$, arterial $\mathrm{pH}(\mathrm{P}=0.01)$, and serum lactate level $(\mathrm{P}<0.001)$; age, sex, and receiving bystander CPR had no association with favorable outcome [22].

\section{INDICATIONS AND CONTRAINDICATIONS}

We believe that ECPR should only be used in highly selected patients with a cardiac origin of arrest. Furthermore, indications and contraindications may vary according to hospital, experience level of the cardiac arrest team, and readiness of ECLS deployment. To date, there has been a lack of RCTs of ECPR and there are no prospectively validated criteria for EC-

Table 2. ECPR criteria

\begin{tabular}{|c|c|}
\hline Favorable criteria & Unfavorable criteria \\
\hline \multicolumn{2}{|l|}{ Witnessed collapse \& bystander CPR (no-flow time $<5$ minutes) } \\
\hline Age $<75$ years & Age $\geq 75$ years \\
\hline \multicolumn{2}{|l|}{ Initially shockable rhythm } \\
\hline $\begin{array}{l}\text { No sustained ROSC within } 15 \text { minutes of ACLS (short low- } \\
\text { flow time }<60 \text { minutes) }\end{array}$ & $\begin{array}{l}\text { Prolonged CPR }>20 \text { minutes in the case of asystole (exceptions: accidental hypo- } \\
\text { thermia, intoxication, near-drowning, and suspected pulmonary embolism) or } \\
\geq 120 \text { minutes in the case of persistent VF/VT }\end{array}$ \\
\hline Presumed correctable causes, especially cardiac etiology & Cardiac arrest due to a clearly uncorrectable cause or trauma \\
\hline \multirow[t]{6}{*}{ High-quality $\mathrm{CPR}\left(\mathrm{ETCO}_{2} \geq 10 \mathrm{~mm} \mathrm{Hg}\right)$} & Low-quality CPR $\left(\mathrm{ETCO}_{2}<10 \mathrm{~mm} \mathrm{Hg}\right)$ \\
\hline & Clinical signs of severe irreversible brain damage or poor neurological prognosis \\
\hline & $\begin{array}{l}\text { Presence of a terminal illness, malignancy, or comorbidity with reduced life expec- } \\
\text { tancy }\end{array}$ \\
\hline & Patient refusal \\
\hline & No informed consent from the family \\
\hline & Low pH (<6.8) or high lactate level $(\geq 20 \mathrm{mmol} / \mathrm{L})$ \\
\hline
\end{tabular}

ECPR: extracorporeal cardiopulmonary resuscitation; CPR: cardiopulmonary resuscitation; ROSC: return of spontaneous circulation; ACLS: Advanced Cardiac Life Support; VF: ventricular fibrillation; VT: ventricular tachycardia; $\mathrm{ETCO}_{2}$ : end-tidal expiratory pressure of carbon dioxide. 
PR indications or patient selection. However, favorable outcome can be expected with ECPR when employed for cardiac arrest under several conditions (Table 2) [23,24]. In the real world, ECPR is commonly performed and quite useful in the cardiac surgical ICU, medical cardiac ICU, and cardiac catheterization laboratory. ECPR is also generally indicated in patients who experience cardiac arrest before or after cardiac surgery or intervention. When a cardiac diagnosis appears to be irreversible, cardiac replacement therapy, such as heart transplantation or artificial heart, should be considered.

Patients who experience witnessed collapse and receive immediate bystander CPR are the ideal target for ECPR. The no-flow time, defined as the duration of cardiac arrest before $\mathrm{CPR}$, is considered to be the main variable that determines neurological prognosis. Although the effect of bystander CPR failed to show statistical significance in a meta-analysis (OR, 2.81; $95 \% \mathrm{CI}, 0.95$ to $8.32, \mathrm{P}=0.06$ ) [22], there was substantial interstudy heterogeneity and lack of robustness.

Since advanced age in OHCA has been associated with low probability of survival [25], many institutions only implement ECPR in cardiac arrest patients under 75 years of age $[13,14,17]$. However, other previous studies have shown that age itself does not have a negative effect on survival after ECPR [15,22]. Thus, advanced age alone should not be considered an absolute contraindication to ECPR. Shockable initial heart rhythm, which includes ventricular fibrillation (VF) and pulseless ventricular tachycardia (VT), is considered a favorable prognostic factor for ECPR $[16,19,21,22]$. A retrospective study of 232 adult patients in OHCA with an initial rhythm of VF or VT found that early ECPR with angiography in the cardiac catheterization laboratory was associated with better neurological outcome at discharge compared with CCPR (42.0\% vs. 15.3\%; OR, 4.0; 95\% CI, 2.08 to 7.7 ; $<<0.001$ ) [26].

As mentioned in the previous section, duration of low-flow time is strongly associated with survival and neurologic outcome in ECPR, especially in patients with initial PEA or shockable rhythm. However, evidence for the appropriate timing of transition from CCPR to ECPR is lacking. According to Ko et al., among patients with IHCA and initial PEA, the best discriminative low-flow time for favorable neurologic outcome was 22 minutes; the corresponding time for those with a shockable rhythm was 46 minutes [19]. A retrospective propensity scorematched study of 156 patients with refractory OHCA evaluated the time of transition to ECPR and showed that mean lowflow time was shorter by 20 minutes when ECPR was initiated after 20 minutes of CCPR compared with ECPR initiation after 30 minutes of CCPR; survival was also better with ECPR initia- tion after 20 minutes of CCPR (29\% vs. $8 \%, \mathrm{P}<0.001)$ [27].

Arterial or venous $\mathrm{pH}$ and serum lactate are important prognostic factors for ECPR [21,22]. However, there is no well-validated cutoff value for either $\mathrm{pH}$ or serum lactate. A high lactate level suggests that a long period of anoxic metabolism has occurred. However, considering that obtaining a level in the peri-arrest period is difficult, its usefulness for selecting patients for ECPR is questionable.

The contraindications for ECPR are few and similar to those for ECLS. They include patients with poor physical activity level, such as those confined to bed; severe permanent neurologic injury; noncardiopulmonary cause of arrest, such as severe sepsis; prolonged CCPR without ROSC; inadequate ACLS, such as failed advanced airway or ineffective chest compression due to severe hypovolemia or unfavorable chest wall anatomy (for example, aortic rupture or severe pectus excavatum); pre-existing severe multiple organ failure; and so on. However, no single one can be considered an absolute contraindication for ECPR; the physician in charge of a patient's care should discuss resuscitation with leaders of the CPR and ECLS teams if the situation arises.

\section{AREA OF UNCERTAINTY AND FUTURE PERSPECTIVES}

Although both ECPR and ELCS are used for patients in cardiac arrest, they are completely different in nature. ECPR is a process that includes CCPR, patient selection, insertion procedure, complication management, post resuscitation care, cause investigation, and cause correction. Much remains to be learned regarding the ECPR process, including indications and proper patient selection.

Controversy over the short-term survival benefit of ECPR over CCPR in patients with OHCA remains $[11,15,16]$; the reason why ECPR seemed not effective is considered due to prolonged low-flow time in OHCA, which is one of the most important prognostic factors for ECPR. Two French studies attempted to reduce low-flow time by performing pre-hospital ECPR on site in selected patients as part of the emergency medical system protocol; early ECPR was initiated after 20 minutes of ACLS $[27,28]$. These trials found that shorter lowflow time improved survival. On the other hand, some centers transport patients in OHCA with refractory VF or VT early to the cardiac catheterization laboratory for percutaneous coronary intervention while performing mechanical CPR until ECPR is started [26]. This strategy improved functionally favorable survival when compared with historical ACLS. 
Although there is currently no high-quality evidence for ECPR, several RCTs are currently underway. The INCEPTION (NCT03101787) and ARREST (NCT03880565) trials are RCTs designed to evaluate the effect of early ECPR initiation in refractory OHCA [29]. The Prague OHCA (NCT01511666) study is a multicenter prospective randomized clinical study investigating the effect of prehospital intra-arrest hypothermia, mechanical chest compression, ECPR, and early invasive investigation and treatment in all patients with OHCA of presumed cardiac origin.

ECPR is a complex intervention that requires a highly trained team, specialized equipment, and multidisciplinary support within a healthcare system. Disastrous complications such as vessel rupture, bleeding, and thromboembolism may occur [5], hence, rigorous training programs should be established. ECPR should only be performed in those patients who might receive benefit. Its indiscreet application could lead to the survival of patients with poor neurological outcome, which may result in considerable patient suffering and family member distress. Therefore, efforts to improve outcome after ECPR should include all individuals involved in the entire process of ECLS care. Future research should be based on such a multidisciplinary approach.

\section{CONCLUSIONS}

ECLS and ECPR are becoming an essential tool in cardiac critical care, cardiac surgery, and cardiac intervention. However, to generalize their use, improved understanding and implementation are required. The studies conducted to date have shown that better survival and neurological outcome can be expected in IHCA patients who receive ECPR compared with CCPR; however, a short-term survival benefit has not yet been shown in OHCA patients.

Prognostic factors associated with better survival and neurological outcome include shorter low-flow time, initial shockable rhythm, lower lactate level, higher $\mathrm{pH}$, and lower SOFA score. Although there are no clearly defined indications, most centers perform ECPR for young patients with an initial shockable rhythm or presumed correctable cause and those with a witnessed collapse and bystander CPR without ROSC within 10-20 minutes of CCPR.

Several recent trials investigating survival after ECPR for OHCA have focused on pre-hospital on-site ECPR and early ECPR with cardiac catheterization. Currently, several RCTs are underway to evaluate the possible benefit of ECPR. However, ECPR is a complex intervention that requires a highly trained team, specialized equipment, and multidisciplinary support and has the risk of potentially fatal complications. Therefore, ECPR should be reserved for carefully selected patients who have the potential to benefit most.

\section{CONFLICT OF INTEREST}

No potential conflict of interest relevant to this article was reported.

\section{ORCID}

Hongsun Kim https://orcid.org/0000-0002-1879-4553

Yang Hyun Cho https://orcid.org/0000-0003-1685-3641

\section{AUTHOR CONTRIBUTIONS}

Conceptualization: all authors. Data curation: HK. Writing original draft: HK. Writing - review \& editing: YHC.

\section{REFERENCES}

1. Centers for Disease Control and Prevention. Out-of-hospital cardiac arrest surveillance: cardiac arrest registry to enhance survival (CARES), United States, October 1, 2005-December 31, 2010. MMWR 2011;60.

2. Peberdy MA, Kaye W, Ornato JP, Larkin GL, Nadkarni V, Mancini ME, et al. Cardiopulmonary resuscitation of adults in the hospital: a report of 14720 cardiac arrests from the National Registry of Cardiopulmonary Resuscitation. Resuscitation 2003;58:297-308.

3. Berdowski J, Berg RA, Tijssen JG, Koster RW. Global incidences of out-of-hospital cardiac arrest and survival rates: systematic review of 67 prospective studies. Resuscitation 2010;81: 1479-87.

4. Kennedy JH. The role of assisted circulation in cardiac resuscitation. JAMA 1966;197:615-8.

5. Annich GM, Lynch WR, MacLaren G, Wilson JM, Bartlett RH. ECMO: extracorporeal cardiopulmonary support in critical care. Seattle: University of Washington Press; 2018.

6. Extracorporeal Life Support Organization. ECLS registry report: international summary [Internet]. Ann Arbor (MI): Extracorporeal Life Support Organization; 2003 [cited 2020 Feb 25]. Available from: https://www.elso.org/Portals/0/Files/Reports/2017/International Summary January 2017.pdf.

7. Richardson AS, Schmidt M, Bailey M, Pellegrino VA, Rycus PT, Pilcher DV. ECMO Cardio-Pulmonary Resuscitation (ECPR), 
trends in survival from an international multicentre cohort study over 12-years. Resuscitation 2017;112:34-40.

8. Extracorporeal Life Support Organization. ECLS registry report: July, 2019 [Internet]. Ann Arbor (MI): Extracorporeal Life Support Organization; 2019 [cited 2020 Feb 25]. Available from: https://www.elso.org/Registry/Statistics/InternationalSummary.aspx.

9. Brooks SC, Anderson ML, Bruder E, Daya MR, Gaffney A, Otto CW, et al. Part 6: alternative techniques and ancillary devices for cardiopulmonary resuscitation: 2015 American Heart Association guidelines update for cardiopulmonary resuscitation and emergency cardiovascular care. Circulation 2015;132 (Suppl 2):S436-43.

10. Panchal AR, Berg KM, Hirsch KG, Kudenchuk PJ, Del Rios M, Cabañas JG, et al. 2019 American Heart Association focused update on advanced cardiovascular life support: use of advanced airways, vasopressors, and extracorporeal cardiopulmonary resuscitation during cardiac arrest: an update to the American Heart Association guidelines for cardiopulmonary resuscitation and emergency cardiovascular care. Circulation 2019;140:e881-94.

11. Kim SJ, Kim HJ, Lee HY, Ahn HS, Lee SW. Comparing extracorporeal cardiopulmonary resuscitation with conventional cardiopulmonary resuscitation: a meta-analysis. Resuscitation 2016;103:106-16.

12. Twohig CJ, Singer B, Grier G, Finney SJ. A systematic literature review and meta-analysis of the effectiveness of extracorporeal-CPR versus conventional-CPR for adult patients in cardiac arrest. J Intensive Care Soc 2019;20:347-57.

13. Chen YS, Lin JW, Yu HY, Ko WJ, Jerng JS, Chang WT, et al. Cardiopulmonary resuscitation with assisted extracorporeal lifesupport versus conventional cardiopulmonary resuscitation in adults with in-hospital cardiac arrest: an observational study and propensity analysis. Lancet 2008;372:554-61.

14. Shin TG, Choi JH, Jo IJ, Sim MS, Song HG, Jeong YK, et al. Extracorporeal cardiopulmonary resuscitation in patients with inhospital cardiac arrest: a comparison with conventional cardiopulmonary resuscitation. Crit Care Med 2011;39:1-7.

15. Maekawa K, Tanno K, Hase M, Mori K, Asai Y. Extracorporeal cardiopulmonary resuscitation for patients with out-of-hospital cardiac arrest of cardiac origin: a propensity-matched study and predictor analysis. Crit Care Med 2013;41:1186-96.

16. Kim SJ, Jung JS, Park JH, Park JS, Hong YS, Lee SW. An optimal transition time to extracorporeal cardiopulmonary resuscitation for predicting good neurological outcome in patients with out-of-hospital cardiac arrest: a propensity-matched study. Crit Care 2014;18:535.
17. Wengenmayer T, Rombach S, Ramshorn F, Biever P, Bode C, Duerschmied D, et al. Influence of low-flow time on survival after extracorporeal cardiopulmonary resuscitation (eCPR). Crit Care 2017;21:157.

18. Lee SW, Han KS, Park JS, Lee JS, Kim SJ. Prognostic indicators of survival and survival prediction model following extracorporeal cardiopulmonary resuscitation in patients with sudden refractory cardiac arrest. Ann Intensive Care 2017;7:87.

19. Ko RE, Ryu JA, Cho YH, Sung K, Jeon K, Suh GY, et al. The differential neurologic prognosis of low-flow time according to the initial rhythm in patients who undergo extracorporeal cardiopulmonary resuscitation. Resuscitation 2020;148:1217.

20. Ryu JA, Chung CR, Cho YH, Sung K, Jeon K, Suh GY, et al. Neurologic outcomes in patients who undergo extracorporeal cardiopulmonary resuscitation. Ann Thorac Surg 2019;108:74955.

21. D’Arrigo S, Cacciola S, Dennis M, Jung C, Kagawa E, Antonelli M, et al. Predictors of favourable outcome after in-hospital cardiac arrest treated with extracorporeal cardiopulmonary resuscitation: a systematic review and meta-analysis. Resuscitation 2017;121:62-70.

22. Debaty G, Babaz V, Durand M, Gaide-Chevronnay L, Fournel E, Blancher M, et al. Prognostic factors for extracorporeal cardiopulmonary resuscitation recipients following out-of-hospital refractory cardiac arrest: a systematic review and metaanalysis. Resuscitation 2017;112:1-10.

23. Riou B, Adnet F, Baud F, Cariou A, Carli P, Combes A, et al. Recommandations sur les indications de l'assistance circulatoire dans le traitement des arrêts cardiaques réfractaires. Ann Fr Anesth Reanim 2009;28:182-6.

24. Michels G, Wengenmayer T, Hagl C, Dohmen C, Böttiger BW, Bauersachs J, et al. Recommendations for extracorporeal cardiopulmonary resuscitation (eCPR): consensus statement of DGIIN, DGK, DGTHG, DGfK, DGNI, DGAI, DIVI and GRC. Clin Res Cardiol 2019;108:455-64.

25. Sulzgruber P, Sterz F, Poppe M, Schober A, Lobmeyr E, Datler P, et al. Age-specific prognostication after out-of-hospital cardiac arrest: the ethical dilemma between 'life-sustaining treatment' and 'the right to die' in the elderly. Eur Heart J Acute Cardiovasc Care 2017;6:112-20.

26. Yannopoulos D, Bartos JA, Raveendran G, Conterato M, Frascone RJ, Trembley A, et al. Coronary artery disease in patients with out-of-hospital refractory ventricular fibrillation cardiac arrest. J Am Coll Cardiol 2017;70:1109-17.

27. Lamhaut L, Hutin A, Puymirat E, Jouan J, Raphalen JH, Jouffroy $\mathrm{R}$, et al. A pre-hospital extracorporeal cardio pulmonary 
resuscitation (ECPR) strategy for treatment of refractory out hospital cardiac arrest: an observational study and propensity analysis. Resuscitation 2017;117:109-17.

28. Lamhaut L, Jouffroy R, Soldan M, Phillipe P, Deluze T, Jaffry M, et al. Safety and feasibility of prehospital extra corporeal life support implementation by non-surgeons for out-of-hos- pital refractory cardiac arrest. Resuscitation 2013;84:1525-9.

29. Bol ME, Suverein MM, Lorusso R, Delnoij TS, Brandon Bravo Bruinsma GJ, Otterspoor L, et al. Early initiation of extracorporeal life support in refractory out-of-hospital cardiac arrest: design and rationale of the INCEPTION trial. Am Heart J 2019; 210:58-68 